\title{
Sampling distant stellar populations on the evolution of galaxies
}

\author{
Arne Ardeberg ${ }^{1}$ and Peter Linde L $^{1,2}$ \\ ${ }^{1}$ Lund Observatory, Lund University, email: arne@astro.lu.se \\ ${ }^{2}$ Malmö University, email: peter@astro.lu.se
}

\begin{abstract}
Modelling and simulation, based on observational data, were used to examine the potential of ELT photometry for studies of the evolution of distant galaxies. An open cluster, a globular cluster and two mixed field populations were employed. Colour-magnitude and metallicity diagrams were examined. For younger populations, excellent turn-off-point age data and abundance data can be obtained even beyond 20Mpc. Higher population age weakens data if not improved with longer exposures. Still, the great potential of ELT photometry for studies of the evolution of galaxies is confirmed. Comments are given on adaptive optics and photometry.
\end{abstract}

Keywords. Galaxies, evolution, ages, abundance data, ELT, star clusters

\section{Introduction}

In spite of large observational and theoretical efforts, the formation and evolution of galaxies remain processes little known. Due to the large distance to the closest substantial sample of all types of galaxies, the Virgo Cluster, not even very large telescopes (VLTs) with adaptive optics (AO) systems can resolve stellar populations adequately for evolutionary studies. This is especially true for the by far most reliable probe of evolution, stellar clusters (Aparicio \& Gallart 2004). With its high photon collection efficiency and high spatial resolution, an extremely large telescope (ELT) with AO can be employed to revolutionise the field, allowing studies of cluster colour-magnitude diagrams (CMDs) and metallicity diagrams (MDs), providing ages and heavy-element abundances for studies of the evolution of distant galaxies. For details, we refer to Ardeberg \& Linde (2004a, 2004b, 2005, 2006a, 2006b) and Linde \& Ardeberg (2006b).

\section{Tools and simulations}

To examine the ELT impact on studies of the evolution of galaxies, we adopted five stellar-population probes, the open cluster NGC 6192, the globular cluster M15 and three mixed field populations. For NGC 6192, we used two simulation versions. The first (as-is) retains the cluster's observed dimensions but doubles its number of stars, to improve our statistics. The other version (extended) represents the same stars as the first version but with linear cluster dimensions increased a factor two. The first version gives a rather crowded open cluster, the second version a more regularly crowded cluster.

Two of the field populations were, like the simulations of NGC 6192 and M15, used as probes of evolution, while the remaining field population was employed as a background field for the two clusters. Placing the probes in a galaxy located at a series of distances from 2 to $20 \mathrm{Mpc}$, we simulated and observed member stars in the Stromgren system with the Euro50 (Ardeberg \& Andersen 2006). Adoption of observed photometry, modelling, simulations, telescope and other simulation parameters are discussed in Ardeberg \& Linde (2004a, 2004b, 2006a) and below. 

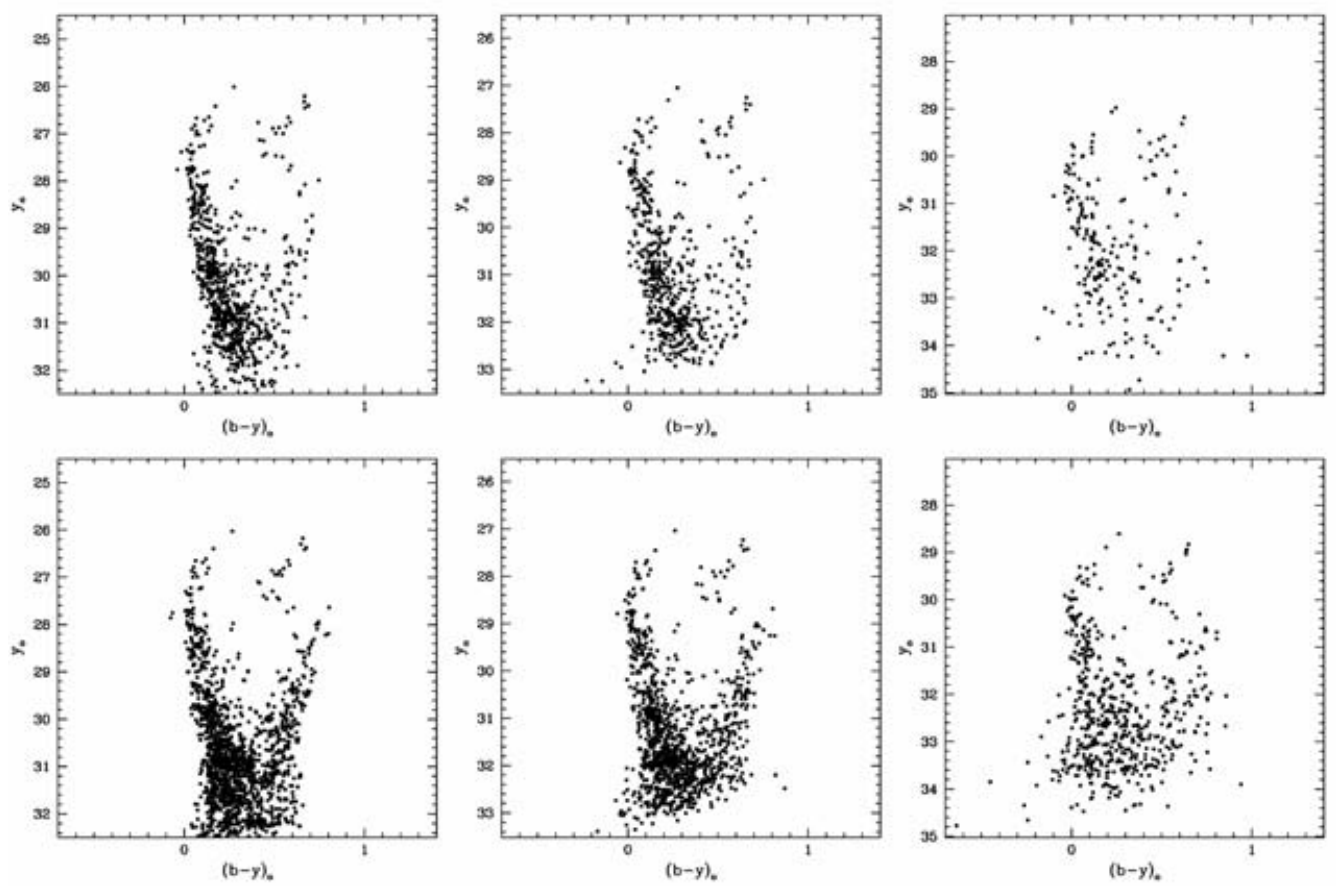

Figure 1. CMDs for the open cluster placed at 5, 8 and $16 \mathrm{Mpc}$ (left to right). Upper boxes represent as-is version, lower boxes the extended version.

\section{Open cluster}

Some of the results obtained from CMD photometry of NGC 6192 are shown in Fig. 1. It shows CMDs for three distances, 5, 8 and $16 \mathrm{Mpc}$. The upper boxes show the results obtained with the as-is version of NGC 6192, the lower boxes corresponding results with the extended version. While completeness, photometric precision and CMD definition in both cases decline with distance, the principal features related to evolution are well maintained over the complete distance interval, confirming the high potential of AO ELT photometry. The advantage of small stellar crowding is clear, notably at large distances.

We used turn-off-point (TOP) data for age estimates. Fig. 1 illustrates the reliability of TOP definition out to 20Mpc. This is confirmed in Fig. 5, showing age estimates versus distance from 2 to $20 \mathrm{Mpc}$. Both cluster versions are included. While the overall results are comparable, the results from the extended cluster are more stable. The difference should be higher for corresponding age data obtained at larger distances. Out to 20Mpc, both sequences show age variations that are very adequate for evolutionary purposes.

To assess the influence of exposure time on age quality, we repeated the procedure for the as-is version of NGC 6192. Instead of 8 hours, we used 4 hours exposures. The data from 8 and 4 hours exposures are given in Fig. 5. While the overall results are similar, the scatter of the long-exposure data is significantly lower than that of the shorter exposures.

In Fig. 2, we show the results from MD photometry of the open cluster. The presentation follows that of Fig. 1 regarding the distances and cluster versions. The data spread increases notably with distance, while the average main-sequence position is maintained. The robustness of the data from the extended version is higher than that from the as-is version. From the MDs, $[\mathrm{Me} / \mathrm{H}]$ data were estimated based on the position of the main sequence. The results are given in Fig. 5, with $[\mathrm{Me} / \mathrm{H}]$ versus distance from 1 to $20 \mathrm{Mpc}$. Both versions show rather reasonable scatter and insignificant trend with distance. The 

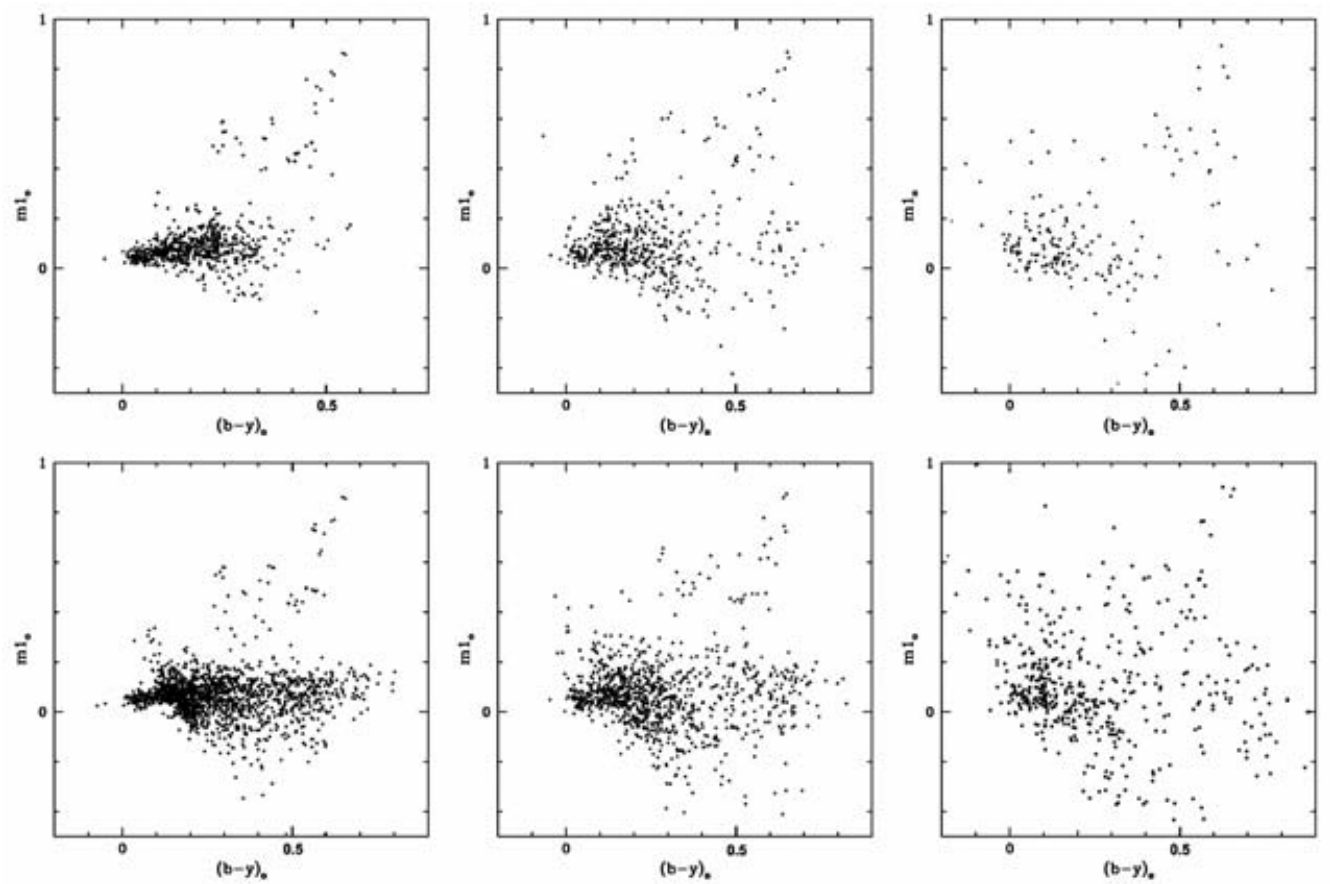

Figure 2. Open cluster MDs for 5, 8 and 16Mpc. Upper and lower boxes as in Fig. 1.

scatter in the extended version is lower than that of the as-is version. Both sequences give favourably low $[\mathrm{Me} / \mathrm{H}]$ variations out to $20 \mathrm{Mpc}$. For the $[\mathrm{Me} / \mathrm{H}]$ data, the prospects for continuation beyond $20 \mathrm{Mpc}$ seem less promising than for the corresponding age data.

\section{Globular cluster}

Three CMDs derived for the 11 Gyr old globular cluster are shown in Fig. 3. They refer to distances 2,5 and $8 \mathrm{Mpc}$. While the $2 \mathrm{Mpc} \mathrm{CMD}$ is very well defined, the influence of distance on CMD scatter is notable. The overall pattern is maintained but sequences get gradually harder to identify. The TOP ages possible to estimate are shown versus distance in Fig. 6. The result confirms the difficulty of TOP definition in CMDs obtained from crowded globular clusters. However, we believe that a stricter discrimination of crowded stars can provide significant help. Corresponding work is in progress.

\section{Mixed field populations}

Three CMDs are, in Fig. 4, given for each mixed field population employed as probe of evolution. The CMDs refer to distances of 5, 8 and $16 \mathrm{Mpc}$. The CMDs of the two younger populations in Fig. 4 are relatively well represented even at $16 \mathrm{Mpc}$. The older populations shown in Fig. 4 lose definition earlier. In both sequences, the younger component is better defined than its older counterpart. In Fig. 6, TOP age estimates are given versus distance for the younger component of each mixed field population, with corresponding data for NGC 6192 as a comparison. The $1 \mathrm{Gyr}$ old population behaves well out to $20 \mathrm{Mpc}$, while the $3 \mathrm{Gyr}$ population shows a more scattered behaviour out to $16 \mathrm{Mpc}$. At larger distances, the TOP cannot be identified for this population. In this case, with longer exposures, TOP identification could probably be extended to $20 \mathrm{Mpc}$. 

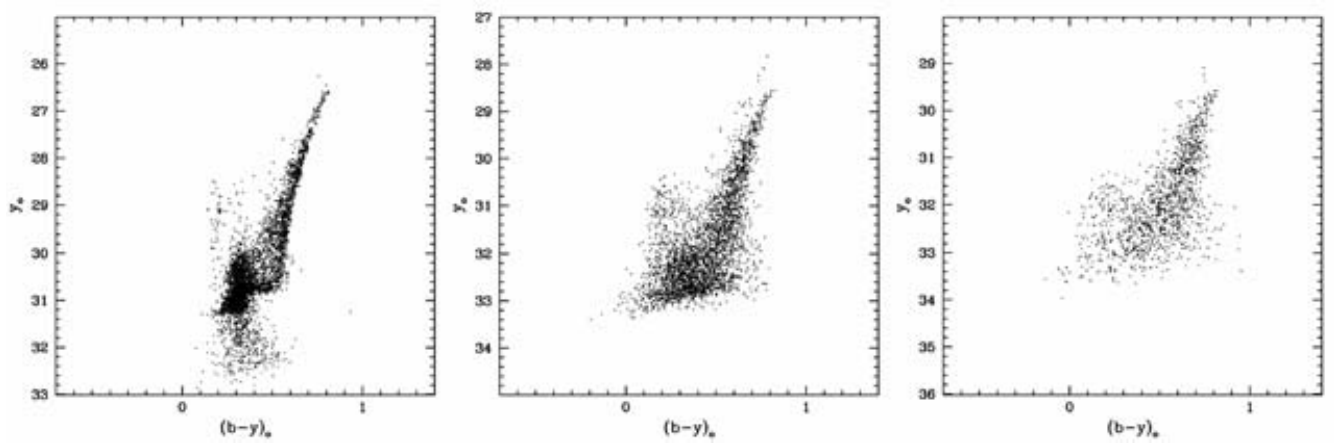

Figure 3. CMDs derived for the globular cluster at 2, 5 and $8 \mathrm{Mpc}$.
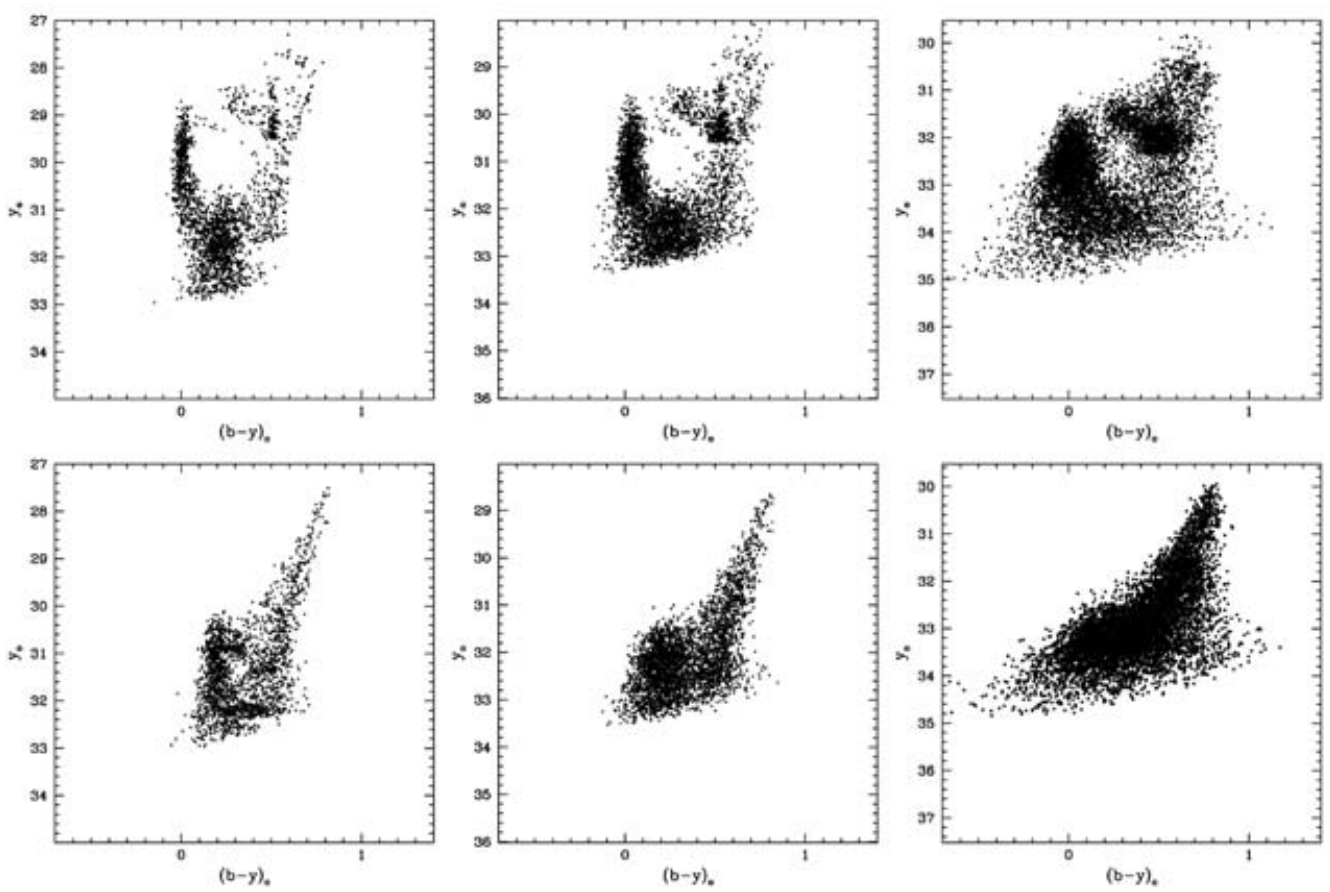

Figure 4. CMDs for mixed-field populations at 5, 8 and 16Mpc. Upper boxes show 1 and 4 Gyr populations, lower boxes 3 and 9 Gyr populations.

For the 4Gyr component in Fig. 4, ages vary systematically with distance over the entire interval, biased by the dominating younger component. For the $9 \mathrm{Gyr}$ component shown in Fig. 4, ages can be estimated only out to $8 \mathrm{Mpc}$ and are uncertain already at smaller distances. Again, the domination of the younger component causes bias.

\section{AO and photometry}

CMDs and MDs are specially relevant if based on optical-visual data. For MDs, this interval is close to necessary for reliable data also for fainter objects. Further, an AO ELT gives images with sharpness dependent on wavelength. See Linde and Ardeberg (2006b). We show that reliable data on galaxy evolution can be obtained from (u)vby photometry. We trust that ELT AO can be developed also for optical-visual wavelengths. Scientific 

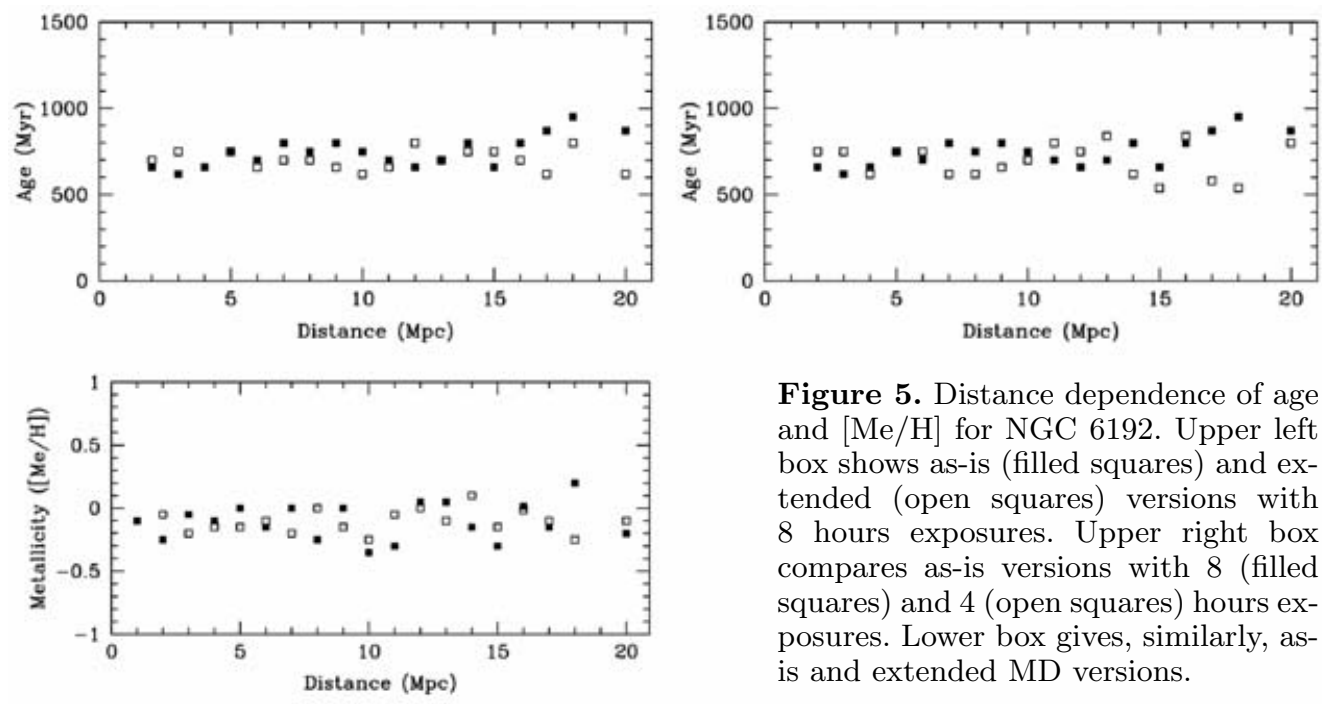

Figure 5. Distance dependence of age and $[\mathrm{Me} / \mathrm{H}]$ for NGC 6192. Upper left box shows as-is (filled squares) and extended (open squares) versions with 8 hours exposures. Upper right box compares as-is versions with 8 (filled squares) and 4 (open squares) hours exposures. Lower box gives, similarly, asis and extended MD versions.
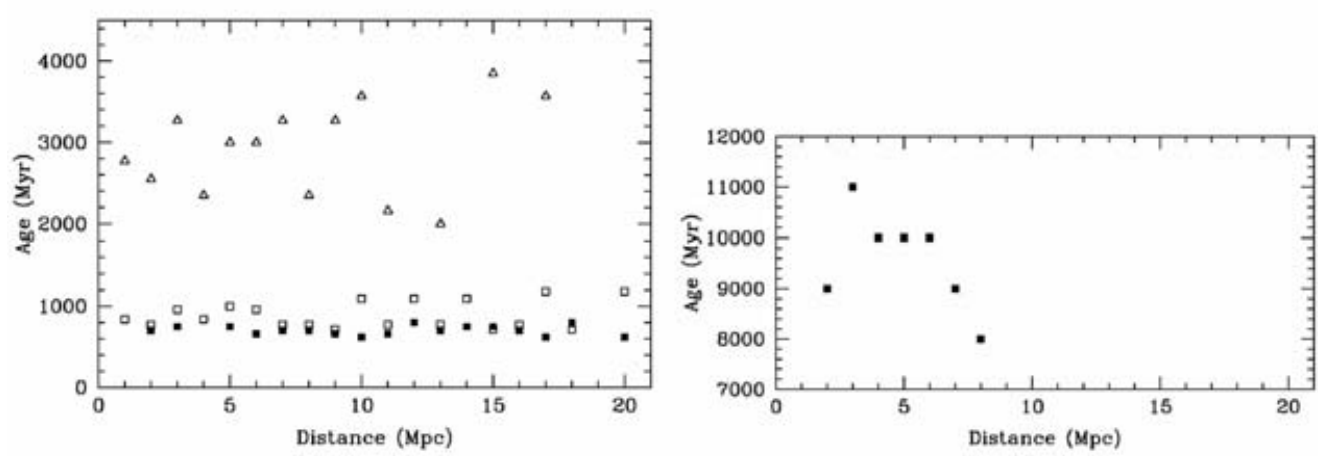

Figure 6. Distance dependence of age estimates. Larger box shows NGC 6192 as-is (filled squares), 1Gyr (open squares) and 3Gyr (triangles) populations. Smaller box gives M15 data.

needs will encourage such development. We anticipate that the technical prerequisites will allow a corresponding AO operation a few years after ELT first light (Ardeberg et al. 2006). On real VLT AO PSFs, we refer to Olsen (2006).

\section{References}

Ardeberg, A. \& Andersen, T. 2006, Proc. JENAM 2004, Granada, in press

Aparicio, A. \& Gallart, C. 2004, AJ 128, 1465

Ardeberg, A. \& Linde, P. 2004b, SPIE 5489, 72

Ardeberg, A. \& Linde, P. 2005, Proc. JENAM 2004, Granada, in press

Ardeberg, A. \& Linde, P. 2006a, in: T. Herbst (ed.), Instrumentation for Extremely Large Telescopes, Ringberg Workshop: http://www.mpia.hd.mpg.de/ELT), in press

Ardeberg, A. \& Linde, P. 2006b, in: T. Herbst (ed.), Instrumentation for Extremely Large Telescopes, Ringberg Workshop: http://www.mpia.hd.mpg.de/ELT), in press

Ardeberg, A., Andersen, T. \& Owner-Petersen, M., 2006, this volume

Linde, P. \& Ardeberg, A. 2006a, T. Herbst (ed.), Instrumentation for Extremely Large Telescopes, Ringberg Workshop: http://www.mpia.hd.mpg.de/ELT), in press

Linde, P. \& Ardeberg, A. 2006b, this volume

Olsen, K. 2006, this volume 


\section{Discussion}

FEAST: At the magnitude at which you are working, is the field population still dominated by stars rather than galaxies?

ARDEBERG: We have looked into this question. Our case is fully dominated by stars with galaxies a relatively minor problem.

ZiJLSTRA: Have you looked at the possibilities of integral field spectroscopy for studying distant clusters?

ARDEBERG: It is on our list of things to do. Spontaneously, I would see roughly three intervals in distance. Out to, and maybe a bit beyond $20 \mathrm{Mpc}$, colour-magnitude and metallicity diagrams provide the best quality data. Further out, integral field spectroscopy is most probably an attractive method, while integral photometry should take care of the largest distances. 